\title{
The Relationship between p53 Expression and Human Papillomavirus in Premalignant and Malignant Uterine Cervical Lesions
}

\author{
Beyhan Varol Mollamehmetoglu1 ${ }^{*}$, Havva Erdem², Muzaffer Keles ${ }^{3}$ \\ ${ }^{1}$ Department of Pathology, Kanuni Training and Research Hospital, Trabzon, Turkey \\ ${ }^{2}$ Department of Pathology, Faculty of Medicine, Ordu University, Ordu, Turkey \\ ${ }^{3}$ Department of Pathology, Faculty of Medicine, Ataturk University, Erzurum, Turkey \\ Email: "bmollamehmetoglu@yahoo.com
}

Received 19 February 2016; accepted 5 April 2016; published 8 April 2016

Copyright (C) 2016 by authors and Scientific Research Publishing Inc.

This work is licensed under the Creative Commons Attribution International License (CC BY).

http://creativecommons.org/licenses/by/4.0/

(c) (i) Open Access

\begin{abstract}
Objective: We aimed to retrospectively examine a series of premalignant and malignant cervical tissues to study a high-risk HPV 16 infection that, among cervical tissue lesions, carries the greatest risk of conversion to cancer, and the presence of p53 protein immunoreactivity, a tumor suppressor gene product. Methods: Paraffin blocks were studied via immunohistochemical (IHC) method to explore the presence of HPV 16 in 59 premalignant and malignant cervical lesions as well as immunoreactivity of the p53 oncoprotein, the most common cellular tumor suppressor gene product in human cancers. Results: In our series, mutant p53 positivity rate was $35.3 \%$ for lowgrade CIL, $\mathbf{4 0 \%}$ for high-grade CIL, and $\mathbf{4 6 . 8 \%}$ for invasive carcinoma cases. Immune staining for high-risk HPV 16 type yielded a positive staining rate of $47 \%$ in low-grade CIL, $80 \%$ in high-grade CIL, and $50 \%$ in invasive carcinoma. Conclusion: The results of our study indicate a progressive increase in p53 oncoprotein reactivity from cervical intraepithelial neoplasia to invasive carcinoma. This suggests the clinical importance of p53 immunoreactivity in dysplastic progression and neoplastic transformation. HPV is the most commonly encountered oncogenic type in cervical lesions, especially in high-grade CIL and invasive carcinomas. Results of the previous reports suggest that HPV-positive carcinomas release wild type p53 and HPV-negative ones release mutant type p53 were not confirmed by our results, which indicated a mutant type p53 reactivity in HPV16 positive carcinoma cases.
\end{abstract}

\section{Keywords}

Cervical Intraepithelial Lesion, Cervix Carcinoma, HPV 16, p53, Immunohistochemistry

\footnotetext{
${ }^{*}$ Corresponding author.
}

How to cite this paper: Mollamehmetoglu, B.V., Erdem, H. and Keles, M. (2016) The Relationship between p53 Expression and Human Papillomavirus in Premalignant and Malignant Uterine Cervical Lesions. Open Journal of Pathology, 6, 73-78. 


\section{Introduction}

Cervix carcinoma is the third most common cancers in women worldwide and most of cases occurring in lowto-medium-resource countries. The introduction of screening programs in many high resource countries has successfully decreased cervical cancer incidence and mortality. Nonetheless, stable or even higher trends have been observed in countries where cervical screening is either absent or of low quality and low coverage [1][3].

Infections by oncogenic high risk (HR) HPV are the most important factor in its etiology; it has been demonstrated that E6 and E7 oncoproteins of HPVs are bound to tumor suppressor genes p53 and Rb and eliminate their tumor suppressing properties, inducing malignancies. At least 13 genotypes of the alpha genus (HPV types $16,18,31,33,35,39,45,51,52,56,58,59$, and 68 ) have been found to be associated with the risk to develop cervical cancer and defined as "carcinogenic" viral types [1]-[5]. HPV typing has been shown to be a criterion determining which cervical intraepithelial neoplasia (CIN) lesions will turn into cancer. HPV16 is the most prevalent genotype in both squamous cell carcinoma (59.3\%) and adenocarcinoma (36.3\%) across the world. There is also a particularly strong correlation between HPV 16 and CIN2-3 lesions.

The likely primary site of HPV infection is transformation zone basal cells accessed via micro-wounds in the epithelial layer. A latent phase infection, in which low-copy number HPV episomers are maintained simply by cell division, shows minimal pathologic features [4]. The productive or permissive phase of infection may show koilocytic and CIN 1. A persistent infection may give rise to the transformative phase characterized by dysregulation of the regular HPV life-cycle and loss of cellular differentiation and maturation resulting in high-grade CIN morphology characterized by basaloid type cell involvement up to the middle to two thirds portion of the lesion (CIN 2) or the full thickness(CIN 3) By HPV typing of cervical biopsies, it is possible to differentiate reactive and neoplastic lesions, to grade morphologically defined CIN lesions, to determine high-grade cases and those who are at risk for the development of cervical carcinoma, and to detect occult and latent HPV infections. Contemporary opinion states that high-risk HPV test would be more efficient when incorporated into a cervical screening program [4] [5].

HR-HPV encodes two early proteins, E6 and E7, that target two major cellular tumor suppressor pathways. E6 targets the p53 tumor suppressor for degradation, result in loss of p53-dependent apoptosis and senescence. E7 binds to the pRb tumor suppressor, thereby disrupting G1/S transition control [1] [3]. Consequently, HRHPV infection may lead to malignant transformation and tumor development.

Herein, we aimed to retrospectively examine a series of premalignant and malignant cervical tissues to study a high-risk HPV 16 infection that, among cervical tissue lesions, carries the greatest risk of conversion to cancer, and the presence of p53 protein immunoreactivity, a tumor suppressor gene product.

\section{Methods}

\subsection{Human Uterine Cervix Samples}

This study included 59 cervical tissue samples sent to Atatürk University Faculty of Medicine, Department of Pathology for examination. The tissue samples consisted of 42 punch biopsies, 14 radical hysterectomy materials, and 3 conization specimens. The histopathological diagnoses of the patients included 17 low-grade cervical intraepithelial lesions (LCIL), 10 high-grade intraepithelial lesions (HCIL), and 32 invasive squamous cell carcinomas. LCIL included CIN1 and koilocyticatypia; HCIL included all CIN2 and CIN3 cases.

\subsection{Immunohistochemistry}

All samples were stained with p53 and HPV 16 antibody using the immunohistochemical method. For staining, 3 - 4 micron thick sections taken from blocks fixated in 10\% formalin and embedded in paraffin were mounted on positive charge microscopic slides. Anti-p53 DO7 antibody, Dako LSAB kit and HPV 16 Ab-2 clone CAM VIR-1 antibody, Biogen Universal kit were used as primary antibody.

\subsection{Immunohistochemical Evaluation}

Cytoplasmic staining was considered positive for HPV 16 whereas only nuclear staining was considered positive for p53. Nuclear staining frequency was graded as none (0), focal (1; staining in $<10 \%$ of epithelial cells), re- 
gional (2; staining in $10 \%-50 \%$ of epithelial cells), and diffuse (3; staining in $>50 \%$ of epithelial cells). These grades were then summated to categorize cervical lesions roughly into three groups with respect to p53: A-lesions with a score of 4 - 6 were graded as having a high level of p53 accumulation; B-lesions with a score of 2 or 3 were graded as having a low level of p53 accumulation; C-lesions with a score of 0 were grades as having no p53 accumulation.

\subsection{Statistical Analysis}

All comparisons were examined for statistical significance using Pearson's Chi-square test with Yates correction and Fischer's exact test (when the number in any category was 5 or less). The threshold for significant $\mathrm{p}$ values was established as $\mathrm{p}<0.05$.

\subsection{Ethics}

The ethics committee of medical faculty hospital approved this study (02.12.2014). As retrospective study, the Ethical Committee does not require the written informed consent from the patients.

\section{Results}

The mean age of the cases was 41 years for LCIL, 47 years for HCIL, and 58 years for invasive carcinoma. Of a total of 59 cases, 25 showed p53 protein expression. Six (35.3\%) of 17 cases with LCIL, 4 (40\%) of 10 cases with HCIL, and 15 (46.8\%) of 32 cases with invasive squamous cell carcinoma showed a positive staining. When high-level p53 accumulation is taken into consideration, there was a significant difference between invasive cancers and high-grade and low-grade intraepithelial lesions with respect to staining $\left(x^{2}=6.44, p=0.009\right.$ and $\mathrm{x}^{2}=7.98, \mathrm{p}=0.012$, respectively). However, there was no significant difference between invasive cancers and HCIL $(\mathrm{p}=0.44)$ (Table 1$)$.

Thirty-two of 59 cases were detected to have HPV 16 antibody. Eight (47\%) of 17 cases with LCIL, 8 (80\%) of 10 cases with HCIL, and 16 (50\%) of 32 cases with invasive squamous carcinoma had positive staining. There was no significant differences between the groups with respect to HPV 16 positivity $(p=0.094, p=0.09$, $\mathrm{p}=0.92)$ (Table 2).

Three of 17 cases (17.6\%) with LCIL had p53 accumulation without having HPV infection. Two (20\%) of 10 cases with HCIL had p53 accumulation. In invasive carcinomas, 7 (21.8\%) of 32 cases had p53 accumulation, of which 5 were high-grade and 2 were low-grade (Table 3).

p53 accumulation with HPV infection was detected in 3 (17.6\%) of 17 cases with LCIL. It was present in 2 (20\%) of 10 cases with HCIL. Eight of 32 invasive cervical carcinoma cases had p53 accumulation (Table 4). No significant correlation was found between HPV infection and p53 positivity rate.

\section{Table 1. p53 accumulation in cervical tissues with immunohistochemical staining.}

\begin{tabular}{ccccc}
\hline Histological diagnosis & Number of cases (n) & \multicolumn{3}{c}{ Number of p53 positive cases } \\
\hline & & Total & Low-grade & High-grade \\
\hline Low-grade CIL & 17 & $6(35.3 \%)$ & $6(35.3 \%)$ & 0 \\
High-grade CIL & 10 & $4(40 \%)$ & 0 & $4(40 \%)$ \\
Invasive carcinoma & 32 & $15(46.8 \%)$ & $5(15.6)$ & $10(31.2 \%)$ \\
\hline
\end{tabular}

Table 2. HPV 16 positivity and negativity rates of the cases.

\begin{tabular}{cccc}
\hline & Number of cases (n) & HPV16 positive & $8(47 \%)$ \\
LCIL & 17 & $8(80 \%)$ & $9(53 \%)$ \\
HICL & 10 & $16(50 \%)$ & $16(50 \%)$ \\
Invasive carcinoma & 32 & $16 \%$ negative & 8 \\
\hline
\end{tabular}


Table 3. p53 accumulation in the absence of HPV infection.

\begin{tabular}{cccccc}
\hline Histological diagnosis & Number of cases (n) & $\begin{array}{c}\text { Number of HPV } \\
\text { Negative cases (N) }\end{array}$ & \multicolumn{2}{c}{ p53 Immunoreactivity } \\
\hline LCIL & 17 & 9 & $6(35.3 \%)$ & $3(17.6 \%)$ & 0 \\
HCIL & 10 & 2 & 0 & 0 & High-grade \\
Invasive carcinoma & 32 & 16 & $9(28.1 \%)$ & $2(6.3 \%)$ & $5(15.6 \%)$ \\
\hline
\end{tabular}

Table 4. p53 accumulation in the presence of HPV infection.

\begin{tabular}{cccccc}
\hline Histological diagnosis & \multicolumn{2}{c}{ Number of cases (n) } & Number of HPV positive cases (N) & \multicolumn{3}{c}{ p53 accumulation } \\
\hline & & & None & Low-grade & High-Grade \\
\hline LCIL & 17 & 8 & $5(29.4 \%)$ & $3(17.6 \%)$ & 0 \\
HCIL & 10 & 8 & $6(60 \%)$ & 0 & $2(20 \%)$ \\
Invasive carcinoma & 32 & 16 & $8(25 \%)$ & $3(9.4 \%)$ & $5(15.6 \%)$ \\
\hline
\end{tabular}

Human papillomavirus infections were also studied in detail using the histological criteria. The typical morphological criteria for HPV infection were studied, which included koilocytosis, perinuclear halo, irregular hyperchromatic nucleus, and dyskeratosis and bi-or multinucleated squamous cells. The comparison of these two methods with each other was shown on Table 5.

\section{Discussion}

Since cervical cancer develops over time and many cervical malignancies originate from progressive malignant conversion of premalignant intraepithelial lesions, CIN lesions emerge at an earlier age than invasive cancer. The mean patient age in each of the three groups in our study support the literature findings [4]-[6]. The morphological criteria of HPV infection were present in $82 \%$ of low-grade CIL and 50\% of high-grade CIL cases. Here, we used the specific and non-specific criteria for HPV infection recommended in the literature. The specific criteria include koilocytosis and dyskeratotic squamous cells. Nonspecific criteria are hypertrophy of squamous cells, bi-or multinucleation, and cytoplasmic amphophilia, clefting, and reddish granulation. Presence of at least three of these nonspecific criteria make the diagnosis of HPV. Koilocytosis has been observed at varying rates of $60 \%, 80 \%$, and $98 \%$ in CIL lesions. Morphological criteria cannot differentiate high-risk HPV subtypes [6] [7]. According to our results, HPV was detected in 6\% of low-grade CIL cases that had no histological criteria in IHC testing, while this rate went up to $40 \%$ in high-grade CIL. This difference may have originated from the presence of undifferentiated cells in superficial layers of epithelium and the absence of koilocytosis in high-grade lesions.

In a large-scale study in Greece, Labropaulou et al. [8] reported a HPV 16 positivity rate of 50\% in cervical cancers, $41 \%$ in HCIL, and 13\% in LCIL. Strand et al. [9], in a study on 2600 women with LCIL, demonstrated a HPV 16 positivity rate of 20\%. It was reported that 22 of 26 LCIL cases that progressed to HCIL were HPV-16 positive. Bergenon et al [10]. detected HPV 16 in 21\% of LCIL cases and 57\% of HCIL cases. Our HPV 16 positivity rate was 50\% in cervical cancer. Jacolien van der Marel et al [11]. demonstrate that HPV 16 is even more etiologically dominant than previously thought, based on various attribution models. Their data show that HPV 16 was the most predominant causal genotype, because in all cases positive for HPV 16 (77\%) by cytology, this genotype was retrieved in whole tissue analysis of the biopsy. The unique role of HPV 16 as a carcinogenic agent has been widely recongnized. The attribution of HPV 16 to cervical cancers, where multiple type infection are far less common than CIN, is over $50 \%$ with a big distance to other carcinogenic types. A Yemelyanova et al. [12] reported 112 contained HPV16 DNA and 32 contained HPV18 DNA of their 150 samples by Q-PCR methods.

Our study found a p53 immunoreactivity rate of 35.3\% in LCIL; 40\% in HCIL; and a total of $46.8 \%$ in invasive carcinoma. Garzetti et al. [13] reported a p53 immunoreactivity rate of 78\% in all CIN lesions studied. Bosari et al. [14] demonstrated a p53 immunoreactivity confined to basal layer in LCILs, while they demonstrated diffuse p53 reactivity at a rate of 25\% in HCIL and 75\% in invasive carcinoma. Akasofu et al. [15] observed no 
Table 5. Histological and immunological detection of HPV infection.

\begin{tabular}{cccccc}
\hline & & \multicolumn{4}{c}{ Histol/IHC } \\
\hline LCIL & $\mathrm{N}$ & ++ & +- & -+ & -- \\
HCIL & 17 & $7(41 \%)$ & $7(41 \%)$ & $1(6 \%)$ & $2(12 \%)$ \\
\hline
\end{tabular}

positivity in CIN 1 and CIN2, while they found a high grade staining of $27 \%$ in CIN3 and $43.5 \%$ in invasive cancer. Holm et al. [16] observed no p53 staining in condyloma, normal and dysplastic tissues, while they showed a p53 imunoreactivity in 7\% of insitu carcinomas and 62\% of invasive squamous cell carcinomas. Li-Di $\mathrm{Xu}$ et al. [1] have examined in their study WIG-1 gene in cervical carcinoma cell lines and Wig-1 expression in both cervical carcinoma cell lines and tumor samples. WIG-1 is a bona fide p53 target gene. Since WIG-1 is a p53 target gene, the question arises as to whether Wig-1 expression levels correlate with p53 status of the and/or presence of HPV, which encodes the E6 protein that targets p53 for degradation. The TP53 gene is more freqently mutated in HPV-negative than HPV-positive cervical cancer cell lines and tumors. Their demonstration that Wig-1 expession levels are higher in HPV-negative cervical carcinoma suggests a possible role of Wig-1 in HPV-negative cervical carcinogenesis.

Many researchers reported that HPV-positive carcinomas release wild-type p53 whereas HPV-negative carcinomas release mutant p53. They showed that E6 protein of oncogenic HPVs have the ability to be bound to p53, a cellular suppressor, and the complex results in inactivation of the suppressor function, causing a decrease in p53 protein. The selective decrease in tumor suppressor protein has been held responsible for cervical carcinogenesis. However, allelic losses and mutations in p53 gene inactivate the tumor suppressing activity and directly contribute to the progression of cellular transformation in HPV-negative cancers. Nevertheless, subsequent studies showed p53 gene mutations also in HPV-positive lesions. The presence of p53 immunoreactivity in HPV 16 positive cases is one of the important findings of our study.

\section{Conclusions}

In conclusion, HPV transmission is increasingly common in young women wordwide and several biological factors may help us to develop more effective preventive screening strategies for detection and treatment of women with cervical carcinomas.

\section{References}

[1] Xu, L.D., Muller, S., Thoppe, S.R., Hellborg, F., Kanter, L., Lerner, M., et al. (2014) Expression of the p53 Target Wig-1 Is Associated with HPV Status and Patient Survival in Cervical Carcinoma. PLoS ONE, 9, e111-e125. http://dx.doi.org/10.1371/journal.pone.0111125

[2] Pinheiro, C., Garcia, E.A., Morais-Santos, F., Scapulatempo-Neto, C., Mafra, A., Streenbergen, R.M.D., et al. (2014) Lactate Transporters and Vascular Factors in HPV-Induced Squamous Cell Carcinoma of the Uterine Cervix. BMC Cancer, 14, 751. http://dx.doi.org/10.1186/1471-2407-14-751

[3] Tornesello, M.L., Buonaguro, L., Giorgi-Rossi, P. and Buonaguro, F.M. (2013) Viral and Cellular Biomarkers in the Diagnosis of Cervical Intraepithelial Neoplasia and Cancer. Biomed Research International, $10 \mathrm{p}$.

[4] Crum, C.P. (1994) Genital Papillomaviruses and Releted Neoplasms: Causation, Diagnosis and Classification (Bethesda). Modern Pathology, 7, 138-145.

[5] Rozendall, L., Walboomers, J.M.M. and Van Der Liden, J.C. (1996) PCR-Based High-Risk HPV Test in Cervical Cancer Screening Gives Objective Risk Assessment of Woman with Cytomorphologically Normal Cervical Smears. International Journal of Cancer, 68, 766-769. http://dx.doi.org/10.1002/(SICI)1097-0215(19961211)68:6<766::AID-IJC13>3.0.CO;2-Z

[6] Kang, S., Jeon, Y.T., Kim, J.W., Park, N.H., Song, Y.S., Kong, S.B. and Lee, H.P. (2005) Polymorphism in the E6 Gene of Human Papillomavirus Type 16 in the Cervical Tissues of Korean Woman. International Journal of Gynecological Cancer, 15, 107-112. http://dx.doi.org/10.1111/j.1048-891x.2005.15010.x

[7] Mclachlin, C.M., Shen, L.H., Sheets, E.E., Kozakewich, H., Perlman, S.E., Tate, J.E. and Crum, C.P. (1997) Disparites in Mean Age and Histopathologic Grade between Human Papillomavirus Type-Spesific Early Cervical Neoplasm. Human Pathology, 28, 1226-1229. http://dx.doi.org/10.1016/S0046-8177(97)90194-5

[8] Labropoulou, V., Diakomanolis, E. and Dailianas, S. (1997) Type-Specific Prevalence of Genital Human Papillomavi- 
ruses in Benign, Premalignant and Malignant Biopsies in Patients from Greece. Sexually Transmitted Diseases, 24, 469-474. http://dx.doi.org/10.1097/00007435-199709000-00005

[9] Strand, A., Wilade, E., Zehbe, I. and Rylander, E. (1997) High Risk HPV Persists after Treatment of Genital Papillomavirus Infection But Not after Treatment of Cervical Intraepithelial Neoplasia. Acta Obstetricia et Gynecologica Scandinavica, 76, 140-144. http://dx.doi.org/10.3109/00016349709050070

[10] Bergeron, C., Barrasso, R., Beaudenon, S., Flamant, P., Croissant, O. and Orth, G. (1992) Human Papillomaviruses Associated with Cervical Intraepithelial Neoplasia: Great Diversity and Distinct Distribution in Low- and High-Grade Lesions. American Journal of Surgical Pathology, 16, 641-649. http://dx.doi.org/10.1097/00000478-199207000-00002

[11] Van Der Marel, J., Quint, W.G.V., Schiffman, M., Van De Sandt, M.M., Zuna, R.E., Terence Dunn, S., et al. (2012) Molecular Mapping of High-Grade Cervical Intraepithelial Neoplasia Shows Etiological Dominance of HPV16. International Journal of Cancer, 131, E946-E953. http://dx.doi.org/10.1002/ijc.27532

[12] Yemelyanova, A., Gravitt, P.E., Ronnett, B.M., Rositch, A.F., Ogurtsova, A., Seidman, J., et al. (2013) Immunohistochemical Detection of Human Papillama Virus Capsid Proteins L1 and L2 in Squamous Intraepithelial Lesions: Potential Utility in Diagnosis and Management. Modern Pathology, 26, 268-274. http://dx.doi.org/10.1038/modpathol.2012.156

[13] Garzetti, G.G., Ciavattini, A., Lucarini, G., Goteri, G., De Nictolis, M., Fabris, G., et al. (1997) p53 Staining and HPV DNA Detection by PCR in Cervical Intraepithelial Neoplasia: Clinical Implications of a Combinated Evaluation. Anticancer Research, 17, 555-560.

[14] Bosari, S., Roncalli, M., Viale, G., Bossi, P. and Coggi, G. (1993) p53 Immunoreactivity in Inflammatory and Neoplastic Diseases of the Uterine Cervix. The Journal of Pathology, 169, 425-430. http://dx.doi.org/10.1002/path.1711690407

[15] Akasofu, M. and Oda, Y. (1995) Immunohistochemical Detection of p53 in Cervical Epithelial Lesions with or without Infection of Human Papillomavirus Types 16 and 18. Virchows Archiv, 425, 593-602. http://dx.doi.org/10.1007/BF00199349

[16] Holm, R., Skomedal, H. and Heliand, A. (1993) Immunohistochemical Analysis of p53 Protein Overexpression in Normal, Premalignant, and Malignant Tissues of the Cervix Uteri. The Journal of Pathology, 169, 21-26. http://dx.doi.org/10.1002/path.1711690105 